\title{
Cingulate metabolites during pain and morphine treatment as assessed by magnetic resonance spectroscopy
}

This article was published in the following Dove Press journal: Journal of Pain Research

19 May 2014

Number of times this article has been viewed

Tine Maria Hansen'

Anne Estrup Olesen ${ }^{2}$

Carsten Wiberg Simonsen'

Asbjørn Mohr Drewes ${ }^{2,3}$

Jens Brøndum Frøkjær'

'Mech-Sense, Department of Radiology, ${ }^{2}$ Mech-Sense, Department of Gastroenterology, ${ }^{3}$ Center for Sensory-Motor Interaction, Department of Health Science and Technology, Aalborg University, Aalborg, Denmark
Correspondence: Jens Brøndum Frøkjær Mech-Sense, Department of Radiology, Aalborg University Hospital, Hobrovej 18-22, 9000 Aalborg, Denmark

Tel +459932 6825

$\mathrm{Fax}+4599326507$

Email jf@mech-sense.com
Background: Experimental investigation of cerebral mechanisms underlying pain and analgesia are important in the development of methods for diagnosis and treatment of pain. The aim of the current study was to explore brain metabolites in response to pain and treatment with morphine.

Methods: Proton magnetic resonance spectroscopy of the anterior cingulate cortex was performed in 20 healthy volunteers (13 males and seven females, aged 24.9 2 2.6 years) during rest and acute pain before and during treatment with $30 \mathrm{mg}$ of oral morphine or placebo in a randomized, double-blinded, cross-over study design. Pain was evoked by skin stimulation applied to the right upper leg using a contact heat-evoked potential stimulator.

Results: Data from 12 subjects were valid for analysis. Painful stimulation induced an increase in $\mathrm{N}$-acetylaspartate/creatine compared with rest $(F=5.5, P=0.04)$. During treatment with morphine, painful stimulation induced decreased glutamate/creatine $(F=7.3, P=0.02)$, myo-inositol/creatine $(F=8.38, P=0.02)$, and $\mathrm{N}$-acetylaspartate/creatine $(F=13.8, P=0.004)$ concentrations, whereas an increase in the pain-evoked $\mathrm{N}$-acetylaspartate/creatine concentration $(F=6.1, P=0.04)$ was seen during treatment with placebo.

Conclusion: This explorative study indicates that neuronal metabolites in the anterior cingulate cortex, such as $\mathrm{N}$-acetylaspartate, glutamate, and myo-inositol, could be related to the physiology of pain and treatment with morphine. This experimental method has the potential to enable the study of brain metabolites involved in pain and its treatment, and may in the future be used to provide further insight into these mechanisms.

Keywords: magnetic resonance imaging, spectroscopy, pain, morphine, anterior cingulate cortex

\section{Introduction}

Knowledge of the pathophysiological changes in chronic pain disorders is important in the development of methods for diagnosis and treatment of pain, including assessment of the effect of analgesic drugs. ${ }^{1}$ Measurement of brain metabolites can be useful to characterize pathologic tissues and diseases. ${ }^{2}$ Proton magnetic resonance spectroscopy $\left({ }^{1} \mathrm{H}-\mathrm{MRS}\right)$ is a noninvasive technique for measurement of metabolite concentrations in vivo such as $\mathrm{N}$-acetylaspartate, glutamate, glutamine, glutamate + glutamine (glx), choline, creatine, myo-inositol, $\gamma$-aminobutyric acid (GABA). MRS has previously been used to assess brain metabolites in studies of chronic pain disorders such as chronic back pain, neuroinflammatory disorders, neuropathic pain and lumbar spine diseases. ${ }^{3-5}$ Experimental MRS studies in healthy subjects have also assessed metabolites in the cingulate cortex and insula during acute pain stimulation. ${ }^{6-10}$ Even though the findings are not completely 
consistent, these studies have shown changes in glutamate, glutamine, glutamate + glutamine (glx), myo-inositol, and GABA concentrations. Glutamate in particular has been shown to increase during acute pain stimulation. ${ }^{6,8-10}$

Pain is often treated with opioids, and MRS has been performed in long-term opiate-dependent subjects. ${ }^{11-13}$ Spectroscopy studies in rats have investigated changes in brain metabolites after chronic treatment with morphine. ${ }^{11-15}$ These studies found changes in the abovementioned brain metabolites as well as others, such as $\mathrm{N}$-acetylaspartate, during treatment with morphine. In rats, many metabolite concentrations decreased after morphine treatment; in particular a decrease in glutamate and $\mathrm{N}$-acetylaspartate have been reported. ${ }^{14}$

To our knowledge, MRS has not been used to assess the effect of morphine treatment on the pain-induced metabolic response in healthy subjects. The anterior cingulate cortex is involved in pain processing as well as being an opioid-rich region of the brain. ${ }^{16-20}$ Therefore, we hypothesized that pain stimulation would increase and morphine-induced analgesia would decrease concentrations of important metabolites in the anterior cingulate cortex. Hence, the aim of the present study was to measure metabolite concentrations in this brain region at rest and in response to acute painful skin heat stimulation before and during treatment with morphine or placebo using a clinical meaningful dose of oral morphine.

\section{Materials and methods Subjects}

Twenty healthy volunteers (13 males, seven females, mean age $24.9 \pm 2.6$ years) participated in this randomized, doubleblinded, placebo-controlled cross-over study. The study was conducted on 2 different days with a minimum washout period of one week between visits. All subjects were scanned prior to the experiment to familiarize them with the scanner environment and to reduce anxiety. The study was carried out in the Department of Radiology, Aalborg University Hospital, Aalborg, Denmark.

All subjects avoided eating and drinking for 4 hours before the experiment. Female subjects were on contraceptives and pregnancy was excluded by a pregnancy test at each visit. Exclusion criteria were abnormal blood pressure, history of previous addictive behavior, history of allergy to opioids, history of pain disorders or mental illness, and intake of analgesic 24 hours prior to the experiment.

The study was approved by the local ethics committee (N-20100046) and the Danish Medicines Agency (2612-4319). The trial was registered at ClinicalTrials.gov (NCT01245244, EUDRACT 2010-020894-17). The study was conducted according to the Declaration of Helsinki and rules of Good Clinical Practice, and monitored by the Good Clinical Practice Unit, Aarhus University Hospital, Denmark.

\section{Drug administration}

Drugs were orally administered as $30 \mathrm{mg}$ of morphine in a $20 \mathrm{~mL}$ solution $(15 \mathrm{~mL}$ of morphine oral liquid mixture $2 \mathrm{mg} / \mathrm{mL}$ and $5 \mathrm{~mL}$ of orange juice concentrate to mask any color or taste), and placebo (15 $\mathrm{mL}$ of placebo solution and $5 \mathrm{~mL}$ of orange juice concentrate) from the hospital pharmacy (Aalborg University Hospital, Aalborg, Denmark). Adverse effects were monitored during the study and one day after the study by telephone interview. MRS data were recorded during rest and during pain stimulation before and 80 minutes after drug administration (see Figure 1).

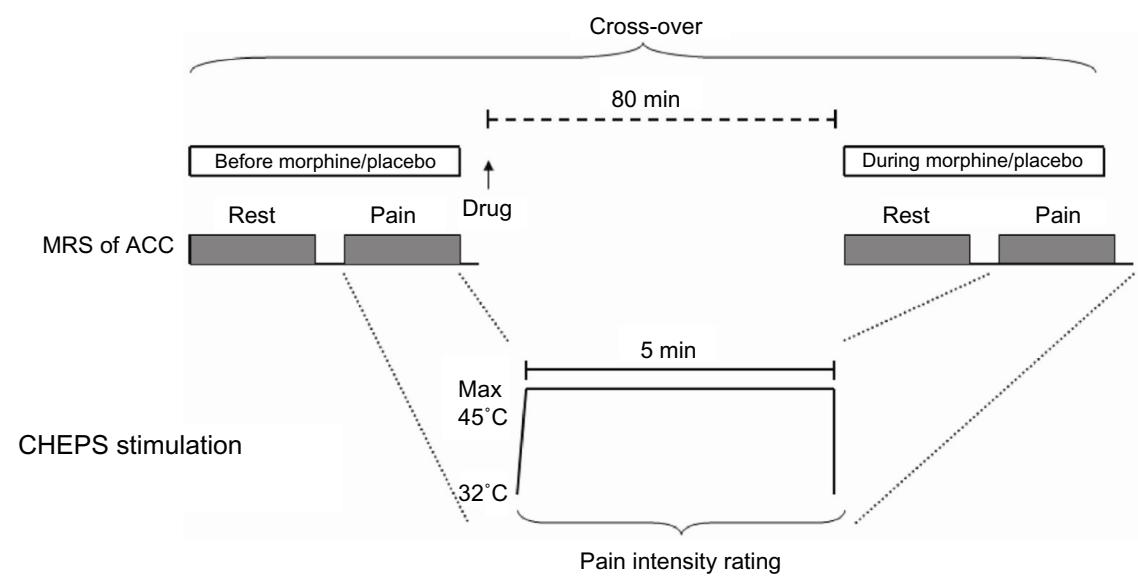

Figure I MRS of ACC was recorded at rest and during painful skin heat stimulation before and 80 minutes after drug administration in a randomized, double-blind, cross-over study using orally administered morphine $(30 \mathrm{mg})$ or placebo. Heat was applied using a contact heat-evoked potential stimulator (CHEPS) for 5 minutes with a destination temperature found prior to the scan (maximum $45^{\circ} \mathrm{C}$ ) and the starting temperature was $32^{\circ} \mathrm{C}$. Subjective pain perception of the stimulus was rated. Abbreviations: ACC, anterior cingulate cortex; MRS, magnetic resonance spectroscopy; CHEPS, contract heat-evoked potential stimulator. 


\section{Experimental acute skin pain stimulation}

Painful skin stimulations were applied $16 \mathrm{~cm}$ proximal to the right knee using a contact heat-evoked potential stimulator (Medoc Ltd, Ramat Yishai, Israel) with an activation area of $573 \mathrm{~mm}^{2}$. A tonic noxious stimulus was applied for 5 minutes at a fixed stimulation temperature, which was found prior to the scan at the first visit for all subjects, hence using the same stimulation temperature for both visits. The starting temperature was $32^{\circ} \mathrm{C}$. To find the stimulation temperature, the stimulus was applied outside the scanner for 30 seconds. The stimulation was stopped if the subject felt medium pain intensity (defined in next section) and the temperature was decreased in steps of $0.5^{\circ} \mathrm{C}$ from $45.0^{\circ} \mathrm{C}$ until the subject tolerated the stimulus temperature for 30 seconds.

During scans, the subjects were able to stop the stimulation in the event of intolerable pain. As the painful stimulation was applied twice, before and during treatment, the thermode was replaced slightly proximal for the second stimulation to avoid repeated stimulation of the same skin area. The stimulation paradigm is shown in Figure 1.

\section{Subjective ratings of pain perception}

Prior to the scan session, the subjects were instructed to rate pain perception on a $0-10$ visual analog scale. After each 5-minute stimulus, subjects were asked to rate the pain level as an average of the entire stimulus. The following anchor words were used to assist in using the visual analog scale: 0 , no sensation; 1 , vague perception of mild sensation; 2 , definite perception of mild sensation; 3 , vague perception of moderate sensation; 4 , definite perception of moderate sensation; 5, pain detection threshold; 6 , slight pain; 7, moderate pain; 8, medium pain; 9 , intense pain; and 10 , unbearable pain. ${ }^{21}$

\section{Imaging data}

Data were acquired on a 3 Tesla GE scanner (General Electric Signa HDxt, GE Healthcare, Milwaukee, WI, USA). A standard eight-channel head coil was used and foam pads fixed the head. Single voxel PRESS (Point RESolved Spectroscopy) MRS was acquired (TR/TE 2,000/30 msec, 2,048 complex points), scan time was 5 minutes, and the total number of scans was 128. Bandwidth was $5,000 \mathrm{~Hz}$. A $20 \times 20 \times 20 \mathrm{~mm}$ voxel of interest was positioned on a sagittal T2-weighted fast spin echo sequence (TR/TE 4,600.0/102.0 msec, matrix $384 \times 256$, slice thickness $3 \mathrm{~mm}$, gap $0.3 \mathrm{~mm}$ ), in the midline in the pregenual ACC with the inferior border along the anterior-posterior commissure line. The position of the voxel of interest is shown in Figure 2. PRESS MRS was also acquired from

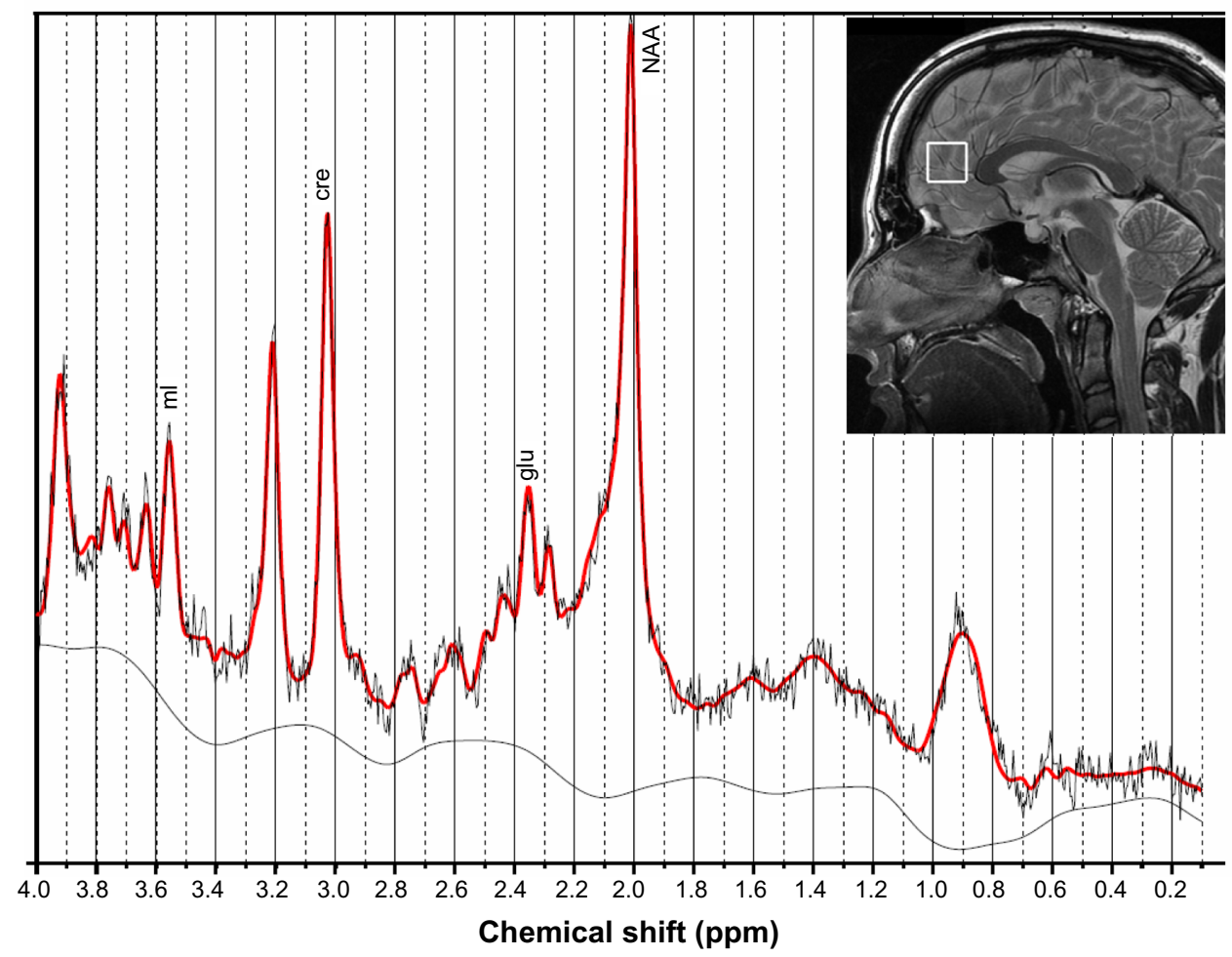

Figure 2 The voxel of interest was placed in the midline in the pregenual anterior cingulate cortex with the inferior border along the anterior-posterior commissure line. An example of the metabolite spectrum is shown, along with peaks for the analyzed metabolites.

Abbreviations: $\mathrm{ml}$, myo-inositol; cre, creatine; glu, glutamine; NAA, N-acetylaspartate. 
a phantom after each experiment to monitor for potential scanner drift.

\section{Imaging processing and statistical analysis}

Spectroscopy data were analyzed using the LCModel method (version 6.2-0X). ${ }^{22}$ Water scaling and eddy current correction was done. We investigated the metabolites $\mathrm{N}$-acetylaspartate, glutamate, and myo-inositol in ratios to creatine concentration, which all had sufficient quality, with a Cramér-Rao $<20 \%$. The Cramér-Rao is a reliability measurement and is the estimated standard deviation in percent of the estimated concentration. ${ }^{23}$ Metabolite concentrations were measured as $\mathrm{mM}$ and ratios were calculated from metabolite concentrations. Figure 2 shows an example of the metabolite spectrum. Metabolites were fitted in the chemical shift range of $0.1-4.0 \mathrm{ppm}$.

One-way repeated measurement analysis of variance was done in SigmaStat 3.0 (SPSS Inc., Chicago, IL, USA) to test for differences in metabolite concentrations and pain intensity ratings. Pain intensity during treatment was also calculated as change compared with ratings before treatment (during treatment/before treatment $\times 100 \%$ ). Data are presented as the mean \pm standard deviation. The significance level was set at $P=0.05$.

\section{Results}

All 20 subjects completed the study. Some scans were excluded due to movement, artifact, and equipment malfunction.
Hence, the total number of included subjects with complete data before treatment was 12 . Of these 12 subjects, eleven had complete data during morphine treatment and nine had complete data during placebo treatment. Full width at half maximum, signal-to-noise ratio, and Cramér-Rao are shown in Table 1.

\section{Skin stimulation temperature and pain perception}

The stimulation intensity was $44.9^{\circ} \mathrm{C} \pm 0.7^{\circ} \mathrm{C}$ on the morphine day and $44.7^{\circ} \mathrm{C} \pm 0.4^{\circ} \mathrm{C}$ on the placebo day $(F=1.8$, $P=0.21)$. The pain intensity ratings were $6.8 \pm 1.0$ before and 6.3 \pm 1.2 during morphine treatment $(F=4.2, P=0.07)$ and $6.5 \pm 1.1$ before and $6.7 \pm 1.1$ during placebo treatment $(F=0.5, P=0.51$, see Table 1$)$. The pain intensity rating decreased to $91.5 \% \pm 10.9 \%$ during morphine treatment and was unchanged during placebo treatment $(100.1 \% \pm 7.8 \%)$ compared with ratings before treatments, but no significant difference was seen between the morphine and placebo treatments $(F=2.8, P=0.14)$.

\section{Pain-induced effect on cingulate metabolites}

Table 1 shows the metabolite concentrations. No significant difference was seen for any metabolite concentration between the 2 days during rest (all $P>0.05$ ). Hence, data from the 2 days were taken into account and the means of the metabolite concentrations for the 2 days were calculated

Table I Overview of the means \pm standard deviations of quality measurements (full width at half maximum, signal-to-noise ratio, Cramér-Rao), pain ratings, and metabolite concentrations in the anterior cingulate cortex

\begin{tabular}{|c|c|c|c|c|c|c|}
\hline & \multicolumn{2}{|l|}{ Rest } & \multirow{2}{*}{\multicolumn{2}{|c|}{$\begin{array}{l}\text { Acute pain stimulation } \\
\text { before treatment }\end{array}$}} & \multirow{2}{*}{\multicolumn{2}{|c|}{$\begin{array}{l}\text { Acute pain stimulation } \\
\text { during treatment }\end{array}$}} \\
\hline & \multirow{2}{*}{$\begin{array}{l}\text { Morphine } \\
(n=12)\end{array}$} & \multirow{2}{*}{$\begin{array}{l}\text { Placebo } \\
(n=12)\end{array}$} & & & & \\
\hline & & & $\begin{array}{l}\text { Morphine } \\
(n=12)\end{array}$ & $\begin{array}{l}\text { Placebo } \\
(n=12)\end{array}$ & $\begin{array}{l}\text { Morphine } \\
(n=I I)\end{array}$ & $\begin{array}{l}\text { Placebo } \\
(n=9)\end{array}$ \\
\hline FWHM & $0.045 \pm 0.012$ & $0.042 \pm 0.011$ & $0.046 \pm 0.013$ & $0.046 \pm 0.010^{*}$ & $0.046 \pm 0.017$ & $0.045 \pm 0.013$ \\
\hline SNR & $14.2 \pm 5.7$ & $14.8 \pm 5.5$ & $14.8 \pm 3.8$ & $14.6 \pm 3.0 *$ & $13.6 \pm 4.4$ & $13.3 \pm 4.7$ \\
\hline \multicolumn{7}{|l|}{ Cramér-Rao (\%) } \\
\hline cre & $4.3 \pm 2.8$ & $4.1 \pm 1.6$ & $3.7 \pm 0.7$ & $3.8 \pm 1.2$ & $3.9 \pm 1.0$ & $4.2 \pm 2.2$ \\
\hline glu & $9.2 \pm 3.1$ & $9.3 \pm 2.4$ & $8.0 \pm 1.3$ & $8.8 \pm 1.6$ & $9.5 \pm 1.5$ & $9.8 \pm 5.1$ \\
\hline $\mathrm{ml}$ & $6.8 \pm 1.9$ & $6.7 \pm 1.2$ & $6.4 \pm 0.8$ & $6.3 \pm 1.1$ & $6.7 \pm 0.8$ & $7.3 \pm 5.5$ \\
\hline NAA & $4.7 \pm 2.7$ & $4.6 \pm 2.3$ & $3.8 \pm 0.6$ & $4.3 \pm 1.4$ & $4.5 \pm \mathrm{I} .4$ & $4.8 \pm 2.8$ \\
\hline Pain ratings (VAS) & - & - & $6.8 \pm 1.0$ & $6.5 \pm 1.1$ & $6.3 \pm 1.2$ & $6.7 \pm 1.1$ \\
\hline \multicolumn{7}{|l|}{ Metabolite } \\
\hline cre $(\mathrm{mM})$ & $6.80 \pm 0.95$ & $7.03 \pm 0.50$ & $6.78 \pm 0.75$ & $7.04 \pm 0.84$ & $6.77 \pm 0.98$ & $6.80 \pm 0.57$ \\
\hline glu/cre & $1.39 \pm 0.29$ & $1.32 \pm 0.17$ & $1.47 \pm 0.16$ & $1.30 \pm 0.16$ & $1.31 \pm 0.17$ & $1.37 \pm 0.18$ \\
\hline $\mathrm{ml} / \mathrm{cre}$ & $0.84 \pm 0.36$ & $0.83 \pm 0.21$ & $0.84 \pm 0.16$ & $0.82 \pm 0.12$ & $0.80 \pm 0.15$ & $0.79 \pm 0.09$ \\
\hline NAA/cre & $\mathrm{I} .15 \pm 0.07$ & $1.11 \pm 0.12$ & $1.24 \pm 0.12$ & $\mathrm{I} .14 \pm 0.07$ & $\mathrm{I} .14 \pm 0.06$ & $1.19 \pm 0.10$ \\
\hline
\end{tabular}

Notes: *Data from one subject are missing; Cramér-Rao, the estimated standard deviation in percent of the estimated concentration.

Abbreviations: FWHM, full width at half maximum; SNR, signal-to-noise ratio; VAS, visual analog scale; cre, creatine; glu, glutamine; ml, myo-inositol; NAA, $\mathrm{N}$-acetylaspartate. 
for the periods of rest and during painful stimulation before treatment (see Figure 3). During painful stimulation, only $\mathrm{N}$-acetylaspartate/creatine increased significantly compared with rest $(F=5.5, P=0.04)$, while no changes were seen for the other metabolite concentrations (creatine, $F=0.02, P=0.90$; glutamate/creatine, $F=0.3, P=0.62$; myo-inositol/creatine, $F=0.002, P=0.97)$.

\section{Effect of oral morphine/placebo on pain- induced changes in cingulate metabolites}

Measurements of metabolite concentrations during painful stimulation in the morphine arm showed decreased glutamate/ creatine $(F=7.3, P=0.02)$, myo-inositol/creatine $(F=8.4$, $P=0.02)$, and $\mathrm{N}$-acetylaspartate/creatine $(F=13.8, P=0.004)$ compared with painful stimulation before treatment, while creatine concentration was unchanged $(F=0.2, P=0.63)$. On the other hand, only the $\mathrm{N}$-acetylaspartate/creatine ratio ( $F=6.1, P=0.04)$ changed significantly during placebo treatment with a pain-evoked increase, while the others were unchanged (creatine, $F=1.0, P=0.36$; glutamate/creatine, $F=1.7, P=0.23$; myo-inositol/creatine, $F=0.7, P=0.41$ ). Plots of $\mathrm{N}$-acetylaspartate/creatine concentrations for individual subjects are shown in Figure 4 for both morphine and placebo treatment. Looking at individual trends of $\mathrm{N}$-acetylaspartate/ creatine changes from before treatment to during treatment, they mostly decreased (ten decreased, one no change) for morphine treatment and increased (seven increased, two decreased) for placebo treatment. To further investigate the mechanisms underlying the observed effect of morphine on brain metabolites, comparisons of metabolite concentration changes induced by the treatment itself without pain stimulation were performed. Comparing metabolite concentrations
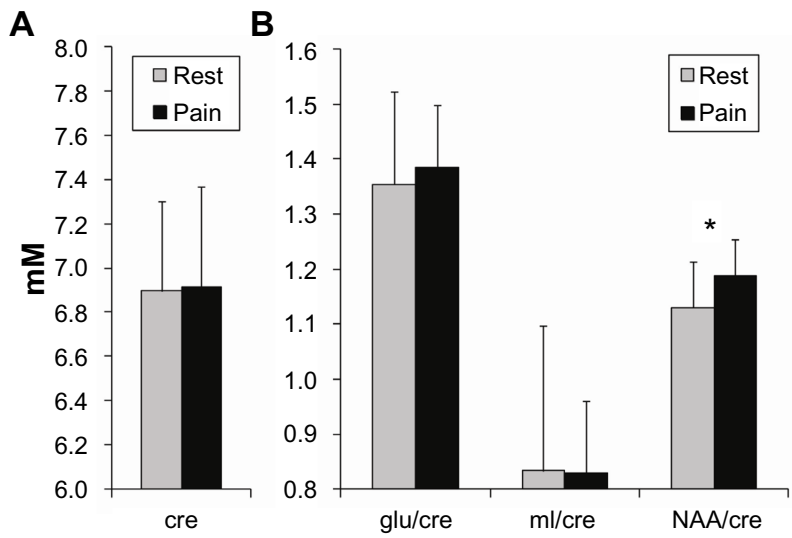

Figure 3 Creatine concentration (A) and metabolite ratios (ratio, no unit) (B) at rest (gray) and during painful stimulus before treatment (black). $* P<0.05$. Abbreviations: $\mathrm{ml}$, myo-inositol; cre, creatine; glu, glutamine; NAA, N-acetylaspartate. during the rest period before morphine treatment with the rest period during morphine treatment, no differences were seen for any the metabolite ratios (glutamate/creatine, myo-inositol/creatine, or $\mathrm{N}$-acetylaspartate/creatine; all $P>0.38$ ). A similar comparison for placebo treatment also showed no differences before and during treatment (glutamate/ creatine, myo-inositol/creatine, or $\mathrm{N}$-acetylaspartate/creatine; all $P>0.15)$.

\section{Discussion}

To our best knowledge, this is the first human explorative study to assess the effect of single-dose oral morphine treatment on the acute pain-induced metabolic response in healthy subjects using in vivo spectroscopy. No difference in metabolite concentrations was observed between the 2 days at rest. Painful skin stimulation induced an increase in $\mathrm{N}$-acetylaspartate/creatine. Treatment with morphine induced decreased concentrations of glutamate/creatine, myo-inositol/creatine, and $\mathrm{N}$-acetylaspartate/creatine during painful stimulation. This is thought to mainly reflect a normalization of the pain-induced increase in $\mathrm{N}$-acetylaspartate/ creatine as seen during placebo treatment. The method seems reliable and may be used to provide further insight into the mechanisms of pain and pain treatment.

\section{Methodological considerations}

The reported changes in brain metabolite concentrations stated in the literature are variable, probably due to variation in the method of pain stimulation, positions of voxels of interest, magnetic field strength, post-processing methods, different patient groups, and ratios compared with absolute concentrations. We analyzed brain metabolites, which have previously been reported to show changes induced by acute pain and opioid treatment. The anterior cingulate cortex is involved in the processing of pain and is also an opioid-rich region. ${ }^{16,18,20}$ We investigated the pregenual anterior cingulate cortex, because this is a region commonly investigated in MRS studies and metabolite changes have previously been reported in acute pain studies. ${ }^{3,7,8}$ However, other parts of the anterior cingulate cortex and brain areas such as the insula would also be relevant to investigate. Chemical shift imaging would be a suitable method for investigating changes in metabolite concentrations in more brain areas at the same time, but the signal-to-noise ratio is normally reduced considerably. ${ }^{2}$ In line with previous studies using spectroscopy, we chose to analyze changes in metabolite concentration as ratios to creatine. ${ }^{5,24}$ Using ratios compared with absolute values of metabolite concentrations are easier to 

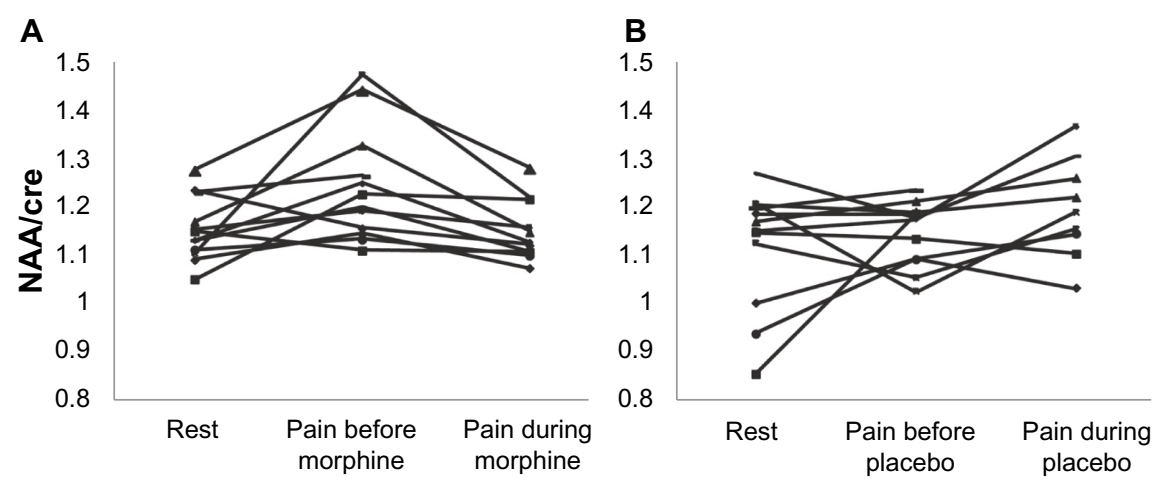

Figure 4 NAA/creatine for each subject during rest and painful stimulation before and during treatment with morphine (A) and placebo (B). Abbreviations: cre, creatine; NAA, $\mathrm{N}$-acetylaspartate.

implement because the computational requirements are less comprehensive and tissue segmentation is not necessary. ${ }^{2} \mathrm{On}$ the other hand, change in absolute quantification of metabolite concentrations is a more direct measurement of changes of a specific metabolite concentration.

Because the study design was placebo-controlled and repeated spectroscopy measurements were included on both the morphine and placebo days, we chose to include only subjects who had complete data before treatment. This was done not to make the statistics too complicated and noncomparable between the different days and treatment conditions. Furthermore, repeated measures analysis of variance would have been most appropriate for analysis of this study design; however, only eight subjects would have been included for analysis, and as this was an explorative study, we wanted to retain as much information from as many subjects as possible so did not use repeated measures analysis of variance.

\section{Effect of morphine on pain perception}

Subjective ratings of pain intensity showed that the stimulus was perceived as slightly to moderately painful and morphine treatment tended to show analgesia. However, compared with placebo, the difference was not significant. It is well known that there is high interindividual variability in analgesic effect and it has been demonstrated that deeper and tonic stimulations are more sensitive to morphine analgesia. ${ }^{25}$

Drug administration in this study was chosen based on our experience from previous studies in which the analgesic effects of this dose in human experimental pain studies were demonstrated. ${ }^{26,27}$ However, it could be hypothesized that a larger dosage could have been more effective at eliciting behavioral and perhaps metabolite changes, but the $30 \mathrm{mg}$ dose is close to the limit we can give in healthy volunteers. Higher doses may lead to adverse effects and high dropout rates. Furthermore, the lack of a significant effect on pain perception during treatment with morphine could be due to the low number of subjects included for analysis. Olesen et al and Staahl et al demonstrated analgesic effects of this dose of morphine in 24 subjects. ${ }^{26,27}$ Therefore, future studies should include more subjects for analysis.

\section{Pain-induced effect on cingulate metabolites}

The $\mathrm{N}$-acetylaspartate/creatine ratio increased significantly in response to acute painful stimulation. The exact physiological mechanisms responsible for the changes in brain metabolite concentrations in response to acute pain are not known. $\mathrm{N}$-acetylaspartate is well known to be a neuronal and axonal marker and involved in neuronal metabolism. ${ }^{28-32} \mathrm{~N}$-acetylaspartate is synthesized within the mitochondria, and decreased concentrations of $\mathrm{N}$-acetylaspartate are correlated with a reduction in adenosine triphosphate and oxygen consumption. ${ }^{29,32}$ We showed an increased $\mathrm{N}$-acetylaspartate/creatine ratio in response to acute pain, which might be explained by the role of $\mathrm{N}$-acetylaspartate in neuronal metabolism. Clark et al proposed $\mathrm{N}$-acetylaspartate to be a reservoir of glutamate. ${ }^{30}$ Hence, changes in $\mathrm{N}$-acetylaspartate and glutamate could be considered to be related in a complex metabolic way, explaining why acute pain may induce changes in the total $\mathrm{N}$-acetylaspartate/glutamate balance. Theoretically, this could explain the observation of changes in glutamate in some studies and $\mathrm{N}$-acetylaspartate in others, depending on the exact setup and conditions. This could also explain why we did not see any pain-induced change in glutamate in the present study. This is consistent with a report by Kupers et al, who investigated metabolite changes in the rostral anterior cingulate cortex following painful tonic heat stimulation. ${ }^{7} \mathrm{On}$ the other hand, Mullins et al reported a painful cold pressor test to evoke an increase in glutamate in the anterior cingulate cortex, and Gussew et al also reported increased glutamate 
concentrations in the anterior insula cortex following painful skin heat stimulation. ${ }^{6,8}$

\section{Effect of morphine on pain-induced changes in cingulate metabolites}

Decreased glutamate/creatine, myo-inositol/creatine, and $\mathrm{N}$-acetylaspartate/creatine were seen on applying painful stimulation during morphine treatment compared with painful stimulation before treatment. In contrast, treatment with placebo increased the concentration of $\mathrm{N}$-acetylaspartate/ creatine. Hence, morphine tends to normalize the concentration to a "rest-like" level, whereas the further increase after placebo treatment could be related to a placebo response. As the metabolite concentrations did not change in response to morphine without pain, this strengthen that the changes in the abovementioned metabolite ratios are actually related to the analgesic effect of morphine.

Overall, the reduction in concentrations of several metabolites, as seen in the present study, is not inconsistent with the results of previous studies in animals and longterm opioid-dependent subjects; however, these results cannot be compared directly with our explorative study of acute pain stimulation during acute morphine treatment. Nuclear magnetic resonance studies have reported changes in metabolite concentrations after chronic morphine treatment in rats. ${ }^{14,15}$ Gao et al found increased levels of GABA and decreased levels of glutamate in the prefrontal cortex, and increased glutamine and myo-inositol levels and decreased GABA, glutamate, Tau, and N-acetylaspartate levels in the hippocampus. ${ }^{14}$ Xiang et al reported an increase in lactate, myo-inositol, and aspartate levels and a decrease in glutamate and glutamine levels in the thalamus, and increased lactate and myo-inositol levels and decreased glutamate levels in the somatosensory cortex. ${ }^{15}$ In both these studies, the concentrations of the different metabolites normalized during the withdrawal period. Other studies investigated long-term opioid-dependent subjects and reported a decreased concentration of $\mathrm{N}$-acetylaspartate, among other changes in metabolite concentrations. ${ }^{11,12}$ Yücel et al showed reduced N-acetylaspartate and glutamate + glutamine levels in the dorsal anterior cingulate cortex in methadone-dependent or buprenorphine-dependent subjects, ${ }^{12}$ and Haselhorst et al investigated metabolic alterations in the frontal lobe in heroin-dependent subjects and reported decreased levels of $\mathrm{N}$-acetylaspartate. ${ }^{11}$ It is not known whether these decreased $\mathrm{N}$-acetylaspartate levels are caused by drug administration or neural changes due to drug dependency. Overall, the results of our study indicate that changes in $\mathrm{N}$-acetylaspartate concentrations are related to the physiology of pain and opioid treatment, and additional changes in glutamate and myo-inositol concentration are also related to morphine treatment.

\section{Conclusion}

In this explorative, placebo-controlled study, we investigated the effect of a single dose of oral morphine on the acute pain-induced metabolic brain response in healthy subjects. We found an increase in $\mathrm{N}$-acetylaspartate/creatine during painful stimulation and a decrease in $\mathrm{N}$-acetylaspartate/creatine during treatment with morphine, which is consistent with previous findings in animal studies and in opioid-dependent subjects, whereas $\mathrm{N}$-acetylaspartate/creatine increased during treatment with placebo. Furthermore, glutamate/creatine and myo-inositol/creatine decreased only during treatment with morphine. The method seems reliable and may in the future be used to provide further insight into the mechanisms of pain and treatment with analgesics.

\section{Acknowledgments}

This study was funded by The Danish Council for Strategic Research and A P Møllers Foundation.

\section{Author contributions}

$\mathrm{TMH}, \mathrm{AEO}$, and JBF: conception and design of the study, acquisition of data, analysis and interpretation of data, drafting the article, revising it critically for important intellectual content, and final approval of the version to be published. CWS: conception and design of the study, acquisition of data, analysis and interpretation of data, revising the paper critically for important intellectual content, and final approval of the version to be published. AMD: conception and design of the study, analysis and interpretation of data, revising the paper critically for important intellectual content, and final approval of the version to be published. All authors discussed the results and commented on the manuscript.

\section{Disclosure}

The authors report no conflicts of interest in this work.

\section{References}

1. Borsook D, Moulton EA, Schmidt KF, Becerra LR. Neuroimaging revolutionizes therapeutic approaches to chronic pain. Mol Pain. 2007;3:25.

2. Jansen JF, Backes WH, Nicolay K, Kooi ME. 1H MR spectroscopy of the brain: absolute quantification of metabolites. Radiology. 2006; 240(2):318-332.

3. Chang L, Munsaka SM, Kraft-Terry S, Ernst T. Magnetic resonance spectroscopy to assess neuroinflammation and neuropathic pain. J Neuroimmune Pharmacol. 2013;8(3):576-593. 
4. Sharma NK, Brooks WM, Popescu AE, et al. Neurochemical analysis of primary motor cortex in chronic low back pain. Brain Sci. 2012;2(3): 319-331.

5. Yabuki S, Konno S, Kikuchi S. Assessment of pain due to lumbar spine diseases using MR spectroscopy: a preliminary report. J Orthop Sci. 2013;18(3):363-368.

6. Gussew A, Rzanny R, Erdtel M, et al. Time-resolved functional 1H MR spectroscopic detection of glutamate concentration changes in the brain during acute heat pain stimulation. Neuroimage. 2010;49(2): 1895-1902.

7. Kupers R, Danielsen ER, Kehlet H, Christensen R, Thomsen C. Painful tonic heat stimulation induces GABA accumulation in the prefrontal cortex in man. Pain. 2009;142(1-2):89-93.

8. Mullins PG, Rowland LM, Jung RE, Sibbitt WL. A novel technique to study the brain's response to pain: proton magnetic resonance spectroscopy. Neuroimage. 2005;26(2):642-646.

9. Gutzeit A, Meier D, Meier ML, et al. Insula-specific responses induced by dental pain. A proton magnetic resonance spectroscopy study. Eur Radiol. 2011;21(4):807-815.

10. Gutzeit A, Meier D, Froehlich JM, et al. Differential NMR spectroscopy reactions of anterior/posterior and right/left insular subdivisions due to acute dental pain. Eur Radiol. 2013;23(2):450-460.

11. Haselhorst R, Dürsteler-MacFarland KM, Scheffler K, et al. Frontocortical $\mathrm{N}$-acetylaspartate reduction associated with long-term IV heroin use. Neurology. 2002;58(2):305-307.

12. Yücel M, Lubman DI, Harrison BJ, et al. A combined spectroscopic and functional MRI investigation of the dorsal anterior cingulate region in opiate addiction. Mol Psychiatry. 2007;12(7):611,691-702.

13. Hermann D, Frischknecht U, Heinrich M, et al. MR spectroscopy in opiate maintenance therapy: association of glutamate with the number of previous withdrawals in the anterior cingulate cortex. Addict Biol. 2012;17(3):659-667.

14. Gao H, Xiang Y, Sun N, et al. Metabolic changes in rat prefrontal cortex and hippocampus induced by chronic morphine treatment studied ex vivo by high resolution $1 \mathrm{H}$ NMR spectroscopy. Neurochem Int. 2007;50(2):386-394.

15. Xiang Y, Gao H, Zhu H, Sun N, Ma Y, Lei H. Neurochemical changes in brain induced by chronic morphine treatment: NMR studies in thalamus and somatosensory cortex of rats. Neurochem Res. 2006;31(10): $1255-1261$

16. Apkarian AV, Bushnell MC, Treede RD, Zubieta JK. Human brain mechanisms of pain perception and regulation in health and disease. Eur J Pain. 2005;9(4):463-484.

17. Jones AK, Qi LY, Fujirawa T, et al. In vivo distribution of opioid receptors in man in relation to the cortical projections of the medial and lateral pain systems measured with positron emission tomography. Neurosci Lett. 1991;126(1):25-28.
18. Petrovic P, Kalso E, Petersson KM, Ingvar M. Placebo and opioid analgesia - imaging a shared neuronal network. Science. 2002; 295(5560):1737-1740.

19. Wagner KJ, Willoch F, Kochs EF, et al. Dose-dependent regional cerebral blood flow changes during remifentanil infusion in humans a positron emission tomography study. Anesthesiology. 2001;94(5): 732-739.

20. Willoch F, Tölle TR, Wester HJ, et al. Central pain after pontine infarction is associated with changes in opioid receptor binding: a PET study with 11 C-diprenorphine. AJNR Am J Neuroradiol. 1999;20(4): 686-690.

21. Drewes AM, Gregersen H, Arendt-Nielsen L. Experimental pain in gastroenterology: a reappraisal of human studies. Scand J Gastroenterol. 2003;38(11):1115-1130.

22. Provencher SW. Estimation of metabolite concentrations from localized in vivo proton NMR spectra. Magn Reson Med. 1993;30(6): 672-679.

23. Provencher SW. Automatic quantitation of localized in vivo $1 \mathrm{H}$ spectra with LCModel. NMR Biomed. 2001;14(4):260-264.

24. Feraco P, Bacci A, Pedrabissi F, et al. Metabolic abnormalities in pain-processing regions of patients with fibromyalgia: a 3T MR. AJNR Am J Neuroradiol. 2011;32(9):1585-1590.

25. Staahl C, Olesen AE, Andresen T, Arendt-Nielsen L, Drewes AM. Assessing analgesic actions of opioids by experimental pain models in healthy volunteers - an updated review. $\mathrm{Br} J$ Clin Pharmacol. 2009;68(2):149-168.

26. Olesen AE, Staahl C, Arendt-Nielsen L, Drewes AM. Different effects of morphine and oxycodone in experimentally evoked hyperalgesia: a human translational study. Br J Clin Pharmacol. 2010;70(2): 189-200.

27. Staahl C, Christrup LL, Andersen SD, Arendt-Nielsen L, Drewes AM. A comparative study of oxycodone and morphine in a multi-modal, tissue-differentiated experimental pain model. Pain. 2006;123(1-2): 28-36.

28. Castillo M, Kwock L, Scatliff J, Mukherji SK. Proton MR spectroscopy in neoplastic and non-neoplastic brain disorders. Magn Reson Imaging Clin N Am. 1998;6(1):1-20.

29. Tsai G, Coylet JT. N-acetylaspartate in neuropsychiatric disorders. Prog Neurobiol. 1995;46(5):531-540.

30. Clark JF, Doepke A, Filosa JA, et al. N-acetylaspartate as a reservoir for glutamate. Med Hypotheses. 2006;67(3):506-512.

31. Moffett JR, Ross B, Arun P, Madhavaroa CN, Namboodiri MA. $\mathrm{N}$-acetylaspartate in the CNS: from neurodiagnostics to neurobiology. Prog Neurobiol. 2007;81(2):89-131.

32. Manji HK, Moore GJ, Rajkowska G, Chen G. Neuroplasticity and cellular resilience in mood disorders. Mol Psychiatry. 2000;5(6): $578-593$.
Journal of Pain Research

\section{Publish your work in this journal}

The Journal of Pain Research is an international, peer-reviewed, open access, online journal that welcomes laboratory and clinical findings in the fields of pain research and the prevention and management of pain. Original research, reviews, symposium reports, hypothesis formation and commentaries are all considered for publication.
Dovepress

The manuscript management system is completely online and includes a very quick and fair peer-review system, which is all easy to use. Visit http://www.dovepress.com/testimonials.php to read real quotes from published authors. 